\title{
BMJ Open Tools to assess the measurement properties of quality of life instruments: a meta-review
}

\author{
Sonia Lorente (D , , ${ }^{1,2}$ Carme Viladrich, ${ }^{1}$ Jaume Vives, ${ }^{1,3}$ Josep-Maria Losilla ${ }^{1,3}$
}

To cite: Lorente S, Viladrich C, Vives J, et al. Tools to assess the measurement properties of quality of life instruments: a meta-review. BMJ Open 2020;10:e036038. doi:10.1136/ bmjopen-2019-036038

\section{- Prepublication history and} additional material for this paper are available online. To view these files, please visit the journal online (http://dx.doi org/10.1136/bmjopen-2019036038).

Received 27 November 2019 Revised 15 June 2020 Accepted 06 July 2020

Check for updates

(C) Author(s) (or their employer(s)) 2020. Re-use permitted under CC BY-NC. No commercial re-use. See rights and permissions. Published by BMJ.

${ }^{1}$ Department of Psychobiology and Methodology of Health Science. Area of Behavioral Science Methodology, Universitat Autònoma de Barcelona, Cerdanyola del Vallés, Barcelona, Spain

${ }^{2}$ Pediatric Area, Consorci Sanitari de Terrassa, Terrassa, Spain

${ }^{3}$ Sport Research Institute, Universitat Autonoma de Barcelona, Cerdanyola, Spain

Correspondence to

Dr Jaume Vives;

Jaume.Vives@uab.cat

\section{ABSTRACT}

Objective This meta-review aims to discuss the methodological, research and practical applications of tools that assess the measurement properties of instruments evaluating health-related quality of life (HRQoL) that have been reported in systematic reviews. Design Meta-review.

Methods Electronic search from January 2008 to May 2020 was carried out on PubMed, CINAHL, PsycINFO, SCOPUS, WoS, Consensus-based Standards for the selection of health Measurement Instruments (COSMIN) database, Google Scholar and ProQuest Dissertations and Theses.

Results A total of 246 systematic reviews were assessed. Concerning the quality of the review process, some methodological shortcomings were found, such as poor compliance with reporting or methodological guidelines. Regarding the procedures to assess the quality of measurement properties, $164(66.6 \%)$ of reviewers applied one tool at least. Tool format and structure differed across standards or scientific traditions (ie, psychology, medicine and economics), but most assess both measurement properties and the usability of instruments. As far as the results and conclusions of systematic reviews are concerned, only $68(27.5 \%)$ linked the intended use of the instrument to specific measurement properties (eg, evaluative use to responsiveness).

Conclusions The reporting and methodological quality of reviews have increased over time, but there is still room for improvement regarding adherence to guidelines. The COSMIN would be the most widespread and comprehensive tool to assess both the risk of bias of primary studies, and the measurement properties of $\mathrm{HRQ}$ oL instruments for evaluative purposes. Our analysis of other assessment tools and measurement standards can serve as a starting point for future lines of work on the COSMIN tool, such as considering a more comprehensive evaluation of feasibility, including burden and fairness; expanding its scope for measurement instruments with a different use than evaluative; and improving its assessment of the risk of bias of primary studies. PROSPERO registration number CRD42017065232.

\section{INTRODUCTION}

The systematic reviews of measurement properties critically appraise the content and measurement properties of all instruments that assess a certain construct of interest in

\section{Strengths and limitations of this study}

The search strategy has been designed to be comprehensive, following the Peer Review of Electronic Search Strategies guidelines including specific filters for finding studies on psychometric properties of measurement instruments.

- A total of 246 systematic reviews were included and, to our knowledge, this meta-review provides the broadest overview of the most common tools used to assess measurement properties of health-related quality of life instruments and their relationship with measurement standards, scientific traditions and the intended use of the measures.

- Some of the included systematic reviews poorly reported the review process, outcomes and conclusions, and this fact may have led to the loss of some data.

- Inclusion of studies published in English only may have led to language bias.

a specific study population. ${ }^{1}$ These systematic reviews provide both a comprehensive overview of the measurement properties of health instruments and supportive evidence for the selection of instruments for a specific purpose (eg, research, clinical practice, predictive). ${ }^{2} 3$ In this type of systematic review, different authors have evaluated not only the methodological quality of their key phases-namely the search strategy, the bias risk assessment of the primary studies and the data synthesis-but also whether the measurement properties of the health status instruments have been appraised with standardised procedures or tools during the data extraction phase. ${ }^{1245}$ However, depending on the measurement standards on which these tools were developed, the approach to analyse the measurement properties of instruments may vary. ${ }^{6}$ This could lead to different conclusions and recommendations, in spite of the effort undertaken by the international Society for Quality of Life Research to set consensus-based minimum standards. ${ }^{7}$ Besides, according to Rosenkoetter and Tate, ${ }^{6}$ 
the assessment tools commonly used by clinicians and researchers to select the appropriate outcome measures for specific purposes show a variety of forms and cover a mix of standards related to reporting, methodological quality and statistical outcome quality.

The aims of this present meta-review are to: (1) identify systematic reviews assessing the measurement properties of health-related quality of life (HRQoL) instruments; (2) identify the main tools applied to assess their measurement properties; (3) describe the contents of the applied tools (validity, reliability, feasibility, etc); (4) identify the measurement standards on which these tools were developed or conform to, comparing their similarities and differences and (5) appraise how authors of these systematic reviews include the assessment of the measurement quality in their results and conclusions, that is, to what extent conclusions depend on the results of the evaluation of the measurement properties, as well as their relationship, if any, with the intended use of the HRQoL instrument (eg, evaluative).

\section{METHODS}

The protocol of this review ${ }^{8}$ was prospectively registered. We conducted this meta-review following the Preferred Reporting Items for Systematic Reviews and Meta-Analyses guidelines (PRISMA). 910

\section{Search strategy}

A systematic search was performed in PubMed, US National Library of Medicine, by National Center for Biotechnology Information (NCBI); CINAHL, Cumulative Index to Nursing and Allied Health Literature, by EBSCOhost; PsycINFO, Psychological Information, by APA PsycNET; SCOPUS by Elsevier; WoS, Web of Science CORE, by Thomson Reuters; Consensus-based Standards for the selection of Health Measurement Instruments database, by COSMIN Initiative (http://www.cosmin. $\mathrm{nl} /$ ); and Google Scholar (up to 400 links). ProQuest Dissertations and Theses Global was used for searching grey literature, and search alerts in all databases were set. The search strategy followed the Peer Review of Electronic Search Strategies guidelines recommendations, ${ }^{11} 12$ and consisted of three filters composed of search terms for the following: (1) systematic review methodology; (2) HRQoL instruments and (3) measurement properties. The latter filter was developed by the Vrije University Medical Center for finding studies on measurement properties of measurement instruments. ${ }^{13}$ All filters were adapted for all databases. The searches were completed in May 2020. Restrictions by language (English) and publication date (from January 2008) were applied (see online supplementary file 1 for search strings for all databases).

\section{Inclusion criteria}

Systematic reviews specifically aiming to report or to assess the measurement properties of instruments evaluating the quality of life within the context of health and disease $^{14}$ were included. Systematic reviews were required to include the full results report, and detailed information about the procedures used to assess the measurement properties.

\section{Exclusion criteria}

Systematic reviews exclusively focused on evaluating clinical interventions were excluded. Systematic reviews specifically focused on assessing patient-reported outcomes measures (PROMs) other than HRQoL for specific diseases, clinical conditions or populations, were excluded. Systematic reviews that did not report full information about the procedures to assess the measurement properties were also excluded (eg, conference abstracts).

\section{Study screening}

References identified by the search strategy were entered to Mendeley reference management software, and duplicates were removed. Titles and abstracts were screened independently by two reviewers (SL and JV). When decisions were unable to be made from title and abstract alone, the full paper was retrieved. Full-text inclusion criteria were checked independently by two reviewers (SL and JV). Discrepancies during the process were resolved through discussion (with independent reviews of J-ML and CV when necessary).

\section{Data extraction}

Extracted information of each selected systematic review and meta-analysis included general information such as author, year and quality of review process of systematic reviews (eg, protocol registration, reporting guidelines and use of flow chart). Information concerning the main identified tools applied to assess the measurement properties of HRQoL instruments included the title, intended use, number of items, response categories, instrument assessment criteria and measurement properties assessed. Information on how authors included the assessment of the quality of HRQoL in their results and conclusions was also extracted. Authors of eligible studies were contacted to provide missing or additional data when necessary.

\section{Study aim}

To examine the methodological, research and practical applications of the reported tools in systematic reviews that assess the measurement properties of instruments evaluating quality of life within the context of health and disease, that is, HRQoL.

\section{RESULTS}

\section{Search results}

Figure 1 shows the results of the search strategy, reported according to the PRISMA flow diagram. A total of 4320 references were identified through database searches. After removing duplicates, 3055 titles and abstracts were screened. After the assessment of 525 full-text documents for eligibility, a total of 246 systematic reviews were included in the qualitative analysis. These systematic 


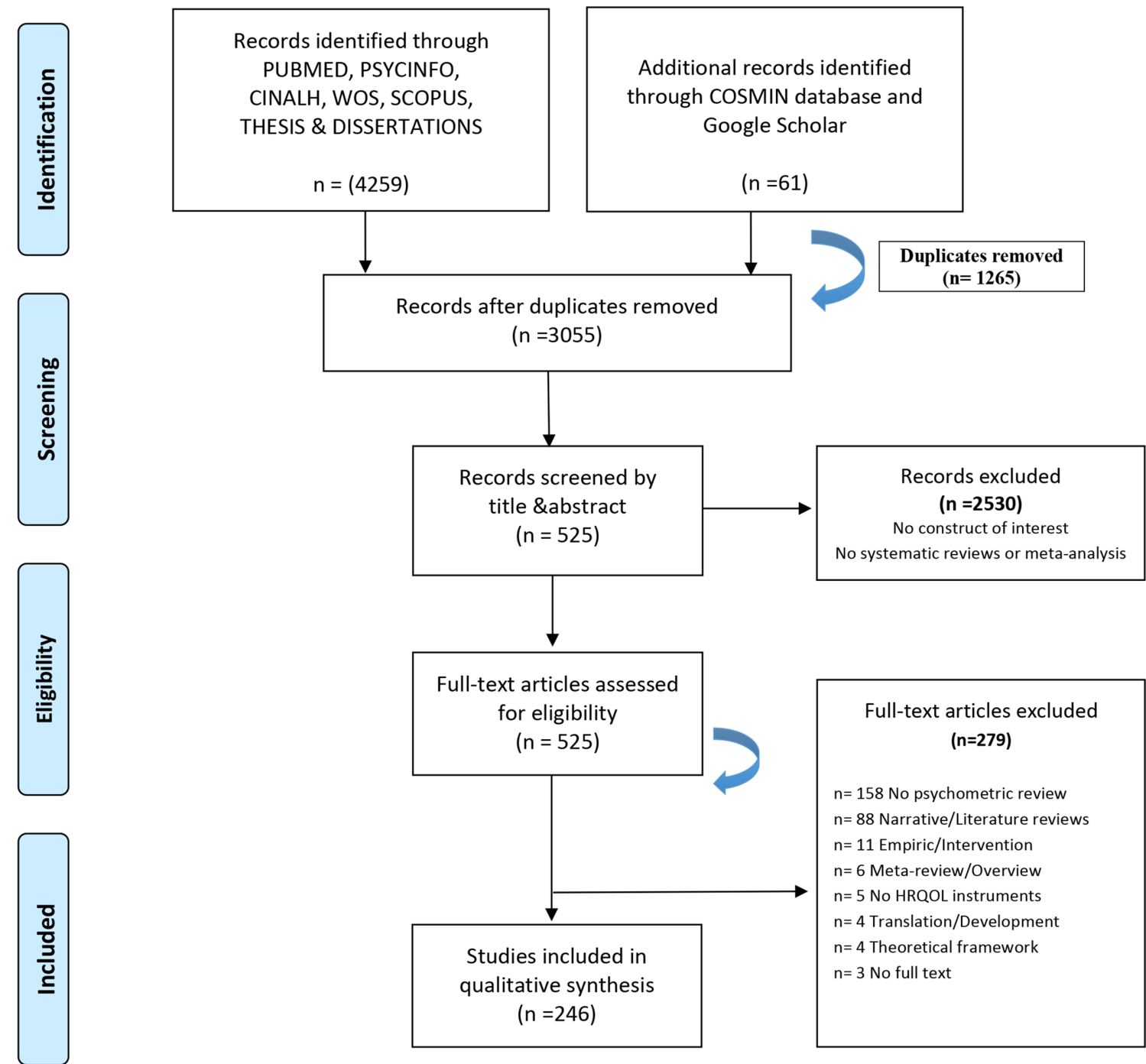

Figure 1 PRISMA flow chart. Flow diagram for search results (from Moher et $a l^{9}$ ). COSMIN, Consensus-based Standards for the selection of health Measurement Instruments; HRQOL, health-related quality of life; PRISMA, Preferred Reporting Items for Systematic Reviews and Meta-Analyses.

reviews covered a wide range of HRQoL instruments, both generic and disease specific. A total of $24(9.8 \%)$ of the systematic reviews assessed the quality of one measurement property only, such as the conceptual and measurement model or the content validity (see online supplementary file S2 for characteristics and references of studies).

\section{Reporting and methodological quality of the studies}

Table 1 shows the reporting and methodological quality of systematic reviews. Findings showed that $27(10.9 \%)$ of the reports registered the protocol prospectively, a figure that raised to $20.8 \%$ when considering the reports from 2014 onwards; 78 (31.7\%) followed reporting guidelines such as PRISMA (50.8\% the last 6 years); 42 (17.0\% since 2008; $23.8 \%$ for the last 6years) assessed the reporting and/or the methodological quality of primary studies using recommended guides, such as Standards for the Reporting of Diagnostic Accuracy Studies and Quality Assessment of Diagnostic Accuracy Studies, respectively;
$238(96.7 \%)$ reported the search strategy; 116 (47.41\%) reported the detailed syntax for one database at least; $134(54.4 \%)$ made the article selection by two or more independent reviewers; $166(67.5 \%)$ used a flow chart to report search outcomes and $132(53.7 \%)$ stated the funding. These last percentages slightly increased when reducing the time frame to the last 6 years.

\section{Assessment of measurement properties of $\mathrm{HRQ}$ oL instruments}

Assessment procedures of measurement properties varied considerably. A total of $164(66.6 \%)$ out of 246 systematic reviews applied one tool at least, that is, a published and well-accepted list of criteria, to rate the evidence on measurement properties of instruments; $41(16.6 \%)$ applied their own author's criteria only; 30 (12.2\%) followed literature recommendations included in very highly circulated books or papers only, and 14 (5.7\%) used an ad hoc checklist of criteria only. A total of $98(39.8 \%)$ systematic reviews did combine different 
Table 1 Reporting and methodological quality of studies

2008-2020 2014-2020

N \% N \%

Protocol registered prospectively

- Yes, PROSPERO

$\begin{array}{llll}27 & 10.9 & 26 & 20.5\end{array}$

No registered

$219 \quad 89.1100$

79.3

Standards of systematic review reporting and/or quality assessment

$\begin{array}{lcccr}\begin{array}{l}\text { Yes (AMSTAR, PRISMA, } \\ \text { QUOROM...) }\end{array} & 78 & 31.7 & 64 & 50.8 \\ \begin{array}{l}\text { - No } \\ \text { Standards to assess reporting and/or quality }\end{array} & 168 \text { assessment of } \\ \text { primary studies } & 68.3 & 62 & 49.2 \\ \text { - Yes (QUADAS, STARD...) } & 42 & 17.0 & 30 & 23.8 \\ \text { - No } & 204 & 83.0 & 96 & 76.2\end{array}$

No of databases searched

$\begin{array}{llrrr}\text { - 1-3 } & 96 & 39.1 & 50 & 39.6 \\ \text { - 4-6 } & 107 & 43.4 & 61 & 48.4 \\ \text { - } \geq 10 & 22 & 8.9 & 8 & 6.3 \\ \text { - Not reported } & 18 & 7.3 & 6 & 4.7 \\ \text { Dt } & 3 & 1.2 & 1 & 0.8\end{array}$

Other sources

$\begin{array}{llrrr}\text { - Official websites/internet } & 25 & 10.1 & 7 & 5.5 \\ \text { - Virtual libraries } & 24 & 9.7 & 12 & 9.4 \\ \text { - Google/google scholar } & 25 & 10.1 & 14 & 11.0 \\ \text { - Scientific journals/thesis } & 6 & 2.4 & 2 & 1.6\end{array}$

Search strategy

Terms, databases, time period

\begin{tabular}{|c|c|c|c|c|}
\hline - Yes & 238 & 96.7 & 123 & 97.6 \\
\hline - No & 8 & 3.3 & 3 & 2.4 \\
\hline \multicolumn{5}{|l|}{ Search syntax } \\
\hline $\begin{array}{l}\text { Detailed syntax reported } \\
\text { (Truncations, Booleans...) }\end{array}$ & 115 & 46.7 & 79 & 62.7 \\
\hline $\begin{array}{l}\text { Syntax not reported or } \\
\text { not detailed enough to be } \\
\text { replicable }\end{array}$ & 125 & 50.8 & 46 & 36.5 \\
\hline $\begin{array}{l}\text { Supplementary file under } \\
\text { request (not available) }\end{array}$ & 5 & 2.1 & 1 & 0.8 \\
\hline \multicolumn{5}{|c|}{ Inclusion/exclusion selection criteria } \\
\hline - Reported and well-defined & 229 & 93.1 & 122 & 96.8 \\
\hline $\begin{array}{l}\text { Not reported or not clearly } \\
\text { stated }\end{array}$ & 17 & 6.9 & 4 & 3.2 \\
\hline
\end{tabular}

Article selection

$\begin{array}{lllll}\text { - By two or more independent } & 134 & 54.4 & 87 & 69.0\end{array}$ reviewers

- Not reported or not clearly $\quad 112 \quad 45.6 \quad 39 \quad 31.0$ stated

Flow chart

\begin{tabular}{lllll} 
- Yes & 166 & 67.5 & 108 & 85.7 \\
\hline
\end{tabular}

Continued
Table 1 Continued

\begin{tabular}{|c|c|c|c|c|}
\hline & \multicolumn{2}{|c|}{$2008-2020$} & \multicolumn{2}{|c|}{ 2014-2020 } \\
\hline & $\mathbf{N}$ & $\%$ & $\mathbf{N}$ & $\%$ \\
\hline - No & 80 & 32.5 & 18 & 14.1 \\
\hline \multicolumn{5}{|l|}{ Funding } \\
\hline - Reported & 132 & 53.7 & 69 & 54.8 \\
\hline $\begin{array}{l}\text { Not reported or not clearly } \\
\text { stated }\end{array}$ & 114 & 46.3 & 57 & 45.2 \\
\hline Total & 246 & 100 & 126 & 100 \\
\hline
\end{tabular}

$\%$, percentage; AMSTAR, assessment of multiple systematic reviews; n, frequency; PRISMA, Preferred Reporting Items for Systematic Reviews and Meta-Analyses; PROSPERO, Prospective Register of Systematic Reviews; QUADAS, Quality Assessment of Diagnostic Accuracy Studies; QUOROM, quality of reporting of meta-analysis; STARD, Standards for the Reporting of Diagnostic Accuracy Studies.

procedures. Most usual combinations were the use of two tools or one tool and literature recommendations.

Tools to assess measurement properties of HRQoL instruments The first 12 columns of table 2 present the characteristics for the identified tools used to assess measurement properties using the last update we are aware of. Tools are reported in order of frequency of use, as pointed out in the last row of the table: (1) 'COSMIN', COSMIN initiative $^{15}{ }^{16}$; (2) 'Quality Criteria for Measurement Properties', Terwee et $a l^{17}$; (3) 'Attributes and Criteria to assess Health Status and Quality of Life Instruments', Scientific Advisory Committee Medical Outcomes Trust $(\mathrm{SACMOT})^{1819}$; (4) 'Health Status Measures in Economic Evaluation', Brazier et $a l^{2021}$; (5) 'Guidance for Industry PROMs', Food and Drug Administration (FDA) ${ }^{22} 23$; (6) 'Evaluating Patient-based Outcomes Measures for use in clinical trials', Fitzpatrick et $a l^{24}$ (also known as Fitzpatrick's criteria); (7) 'International Classification of Functioning' and 'International Classification of Functioning for Children and Youth', $\mathrm{WHO}^{25}$; (8) 'Evaluating Measures of Patient-Reported Outcomes (EMPRO)', Spanish Cooperative Investigation Network for Health and Health Service Outcomes Research ${ }^{26}$; (9) 'Spinal Cord Injury Criteria', Spinal Cord Injury Rehabilitation Evidence $^{27}$ 28; (10) 'Criteria for Assessing the Tools of Disability Outcomes Research', Andresen ${ }^{29}$ (also known as Andresen's tool); (11) 'CanChild Outcomes Measures', CanChild Center for Childhood Disability Research ${ }^{30}$ and (12) 'Outcomes Measures in Rheumatology Clinical Trials (OMERACT)', OMERACT initiative. ${ }^{31}$ Table 2 also includes a final column showing the characteristics of Testing Standards by American Educational Research Association, American Psychological Association and National Council on Measurement in Education ${ }^{32} 33$ (hereinafter 'Testing Standards') initially published in 1954 and regularly updated every decade using consensus based procedures. The Testing Standards are the source of most of the technical vocabulary for measurement 


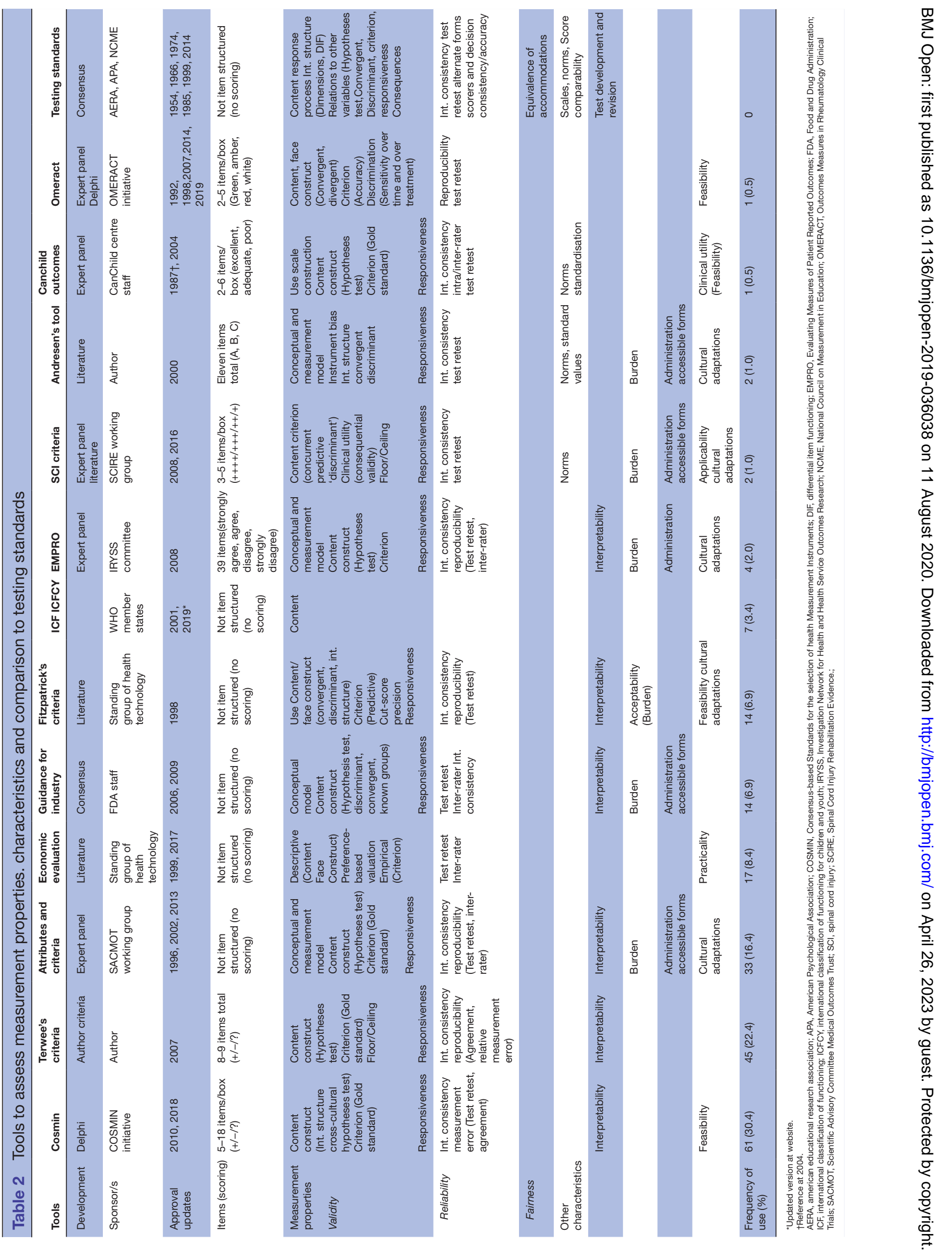


properties in HRQoL instruments, therefore, they will be used as a reference to compare the twelve identified tools. In fact, these standards have already been recommended to establish a unified approach to validity and reliability of results derived from psychometric instruments in clinical medicine, research and education. ${ }^{34}$

Different methodologies were used to develop the tools. The expert panel consensus and the literature review were the most usual methods, led by steering committees or staff/working groups. The format and structure of these tools also vary. Whereas seven of them were itemised to allow the assignment of quality scores, the other six took the form of standards or guidelines. Tools with an itemised structure were the COSMIN, Quality Criteria for Measurement Properties, EMPRO, SCI Criteria, Criteria for Assessing the Tools of Disability Outcomes Research (Andresen's tool), CanChild Outcomes Measures and OMERACT.

Among all measurement properties considered in Testing Standards, 11 out of the 12 tools recommended to assess the conceptual and measurement model; content, structural, convergent, discriminant, concurrent and predictive validity; responsiveness or sensitivity to change; and internal consistency, test-retest and interrater reliability. However, the approach to analyse these measurement properties varied, with examples found in construct validity, criterion validity and reliability. Depending on the tool, the validity of the construct can be evaluated either by hypothesis confirmation in general (eg, COSMIN or EMPRO), or by specific hypothesis based on correlations with other measures, that is, convergent and discriminant validity (eg, Andresen's tool). Criterion validity can be assessed either exclusively by calculating the correlation coefficient with a gold standard (eg, CanChild Outcomes Measures) or by obtaining variously correlation, specificity and sensitivity or predictive values (eg, FDA). Reliability can be analysed either by test retest reliability, inter-rater reliability and internal consistency (eg, FDA), or only by test retest and inter-rater agreement (eg, Economic evaluation). Despite the Testing Standards recommendations, just one tool includes additional criteria to assess consequential validity (SCI), and four assess fairness (eg, accessible forms for subjects with vision impairment or for specific populations) (SACMOT, FDA, SCI and Andresen's tool). None of them includes criteria to assess the validity of response processes. Other HRQoL instrument characteristics, such as feasibility (eg, cost of obtaining a sample), acceptability (eg, suitability from the patient perspective) or burden (eg, the time or effort placed on the administration of the instrument) are assessed instead. Finally, notice that some concepts have changed their place over time. The clearest case is evidence regarding crosscultural equivalence, which was treated as an additional characteristic of the instruments in most tools released before 2014 (eg, EMPRO or SCI), but was considered a proper measurement property in the COSMIN's 2018 update. It is also considered a measurement property in Testing Standards where it is included as a particular case of differential item functioning when assessing the internal structure of the instruments (see online supplementary file S3 for more details).

\section{Intended uses of instruments and their association to measurement properties}

Some of the differences between tools can be attributed to the fact that they are devoted to the evaluation of instruments developed with different intended uses. For instance, COSMIN aims at assessing the quality of instruments for an evaluative purpose whereas the Economic Evaluation tool aims at the assessment of instruments for analytical purposes. Nevertheless, the relation between the intended use of the instruments and the measurement properties assessed is not usually included in the conclusions of the systematic reviews. Table 3 shows the intended use of instruments, based on the framework proposed by McDowell et $a l^{25}$ and the association to measurement properties that reviewers established in their conclusions. The instruments were most frequently used for evaluation $(178,72.3 \%)$ and for assessment of impact of disease on HRQoL (138, 55.1\%), either alone or in conjunction. Other purposes were analytic $(35,14.2 \%)$, diagnostic $(16$, $6.5 \%)$, descriptive $(4,1.6 \%)$ and predictive $(2,0.8 \%)$. A total of $6(2.4 \%)$ systematic reviews did not report or did not clearly state the intended use of the instruments. As far as the assessment and conclusions is concerned, only $68(27.6 \%)$ systematic reviews linked the intended use of the instrument to measurement properties. The most common use was evaluative, generally associated to responsiveness, content validity or reliability, for example. When the purpose was the assessment of the impact of disease on HRQoL, the conceptual and measurement model and content validity were usually reported. The analytical purpose involved reporting preference-based valuation (eg, utility scores) and evidence of agreement, and the diagnostic use was linked to known groups validity and test-retest reliability. To better understand these results, some examples are given. First, the evaluative purpose was associated to responsiveness, we found conclusions such as: 'For use in longitudinal studies or clinical practice, where responsiveness is an issue, the Minnesota Living with Heart Failure Questionnaire and the Chronic Heart Failure Questionnaire would be adequate'. ${ }^{36}$ Second, the intended use was the assessment of the impact of disease on HRQoL, the usual association was to the measurement model and conclusions resembled this one: 'None of the RLS specific QOL measures appears to have been informed by a conceptual model or a conceptual framework. Consequently, none can be considered comprehensive in terms of assessing the full impact of Rest Legs Syndrome on QOL'. ${ }^{37}$ Third, an example illustrating general conclusions, that is, conclusions that did not associate the intended use of the instrument to any specific measurement properties, was as follows: 'None of the available instruments fulfils the 
Table 3 Intended use of instruments and their association to measurement properties

\section{Intended use of instruments identified across the systematic reviews}

\begin{tabular}{|c|c|c|}
\hline $\begin{array}{l}\text { Evaluative (Change scores pre and } \\
\text { poststudies. Effectiveness of an } \\
\text { intervention) }\end{array}$ & 178 & 72.3 \\
\hline $\begin{array}{l}\text { Impact of disease on HRQoL (disease } \\
\text { symptoms, burden...) }\end{array}$ & 138 & 55.1 \\
\hline $\begin{array}{l}\text { Analytic (health policies. Cost- } \\
\text { effectiveness. Funding) }\end{array}$ & 35 & 14.2 \\
\hline $\begin{array}{l}\text { Diagnostic (Distinguish between } \\
\text { groups, levels of severity...) }\end{array}$ & 16 & 6.5 \\
\hline $\begin{array}{l}\text { Descriptive (Health measures in } \\
\text { surveys. Needs of groups of people) }\end{array}$ & 4 & 1.6 \\
\hline $\begin{array}{l}\text { Predictive (Anticipation of future health } \\
\text { status. Risk factors. Risk profiles) }\end{array}$ & 2 & 0.8 \\
\hline $\begin{array}{l}\text { Intended use is no reported or no } \\
\text { clearly stated }\end{array}$ & 6 & 2.4 \\
\hline $\begin{array}{l}\text { Conclusions according to the } \\
\text { intended use of instruments }\end{array}$ & $\mathrm{n}$ & \% (over 246) \\
\hline $\begin{array}{l}\text { Yes, reviewers made specific } \\
\text { conclusions }\end{array}$ & 68 & 27.6 \\
\hline $\begin{array}{l}\text { No, reviewers made general } \\
\text { conclusions }\end{array}$ & 178 & 72.4 \\
\hline $\begin{array}{l}\text { Measurement properties associated } \\
\text { to the intended use of the } \\
\text { instrument }\end{array}$ & n & \% (Over 68) \\
\hline
\end{tabular}

\section{Evaluative}

\begin{tabular}{l}
$\begin{array}{l}\text { Responsiveness/Conceptual and } \\
\text { Measurement Model/Content validity/ }\end{array}$ \\
$\begin{array}{l}\text { Reliability (internal consistency, } \\
\text { test retest)/Respondent Burden/ } \\
\text { Convergent validity/Cross cultural } \\
\text { validity }\end{array}$ \\
$\begin{array}{l}\text { Impact } \\
\quad \begin{array}{l}\text { Conceptual and Measurement } \\
\text { Model/Content validity }\end{array}\end{array}$ \\
$\begin{array}{l}\text { Analytic } \\
\quad \text { Preference-based valuation/ } \\
\text { agreement }\end{array}$ \\
$\begin{array}{l}\text { Diagnostic } \\
\quad \text { Known groups validity/test-retest }\end{array}$ \\
$\begin{array}{l}\text { Predictive } \\
\text { Sensivity and specificity }\end{array}$ \\
\hline
\end{tabular}

(\%), percentage.

HRQoL, health-related quality of life.

psychometric demands of reliability, validity and responsiveness to serve as a primary outcome measure in clinical trials' ${ }^{38}$

\section{DISCUSSION}

The present meta-review identified 246 systematic reviews assessing measurement properties of HRQoL instruments in order to analyse the quality of the review process, describe the most used tools to assess measurement properties and examine how reviewers included the assessment of the quality of HRQoL in their conclusions.

\section{Reporting and methodological quality of the studies}

Findings showed how the reporting and methodological quality of systematic reviews has increased over time. Most reviewers reported the search strategy, stated the inclusion and exclusion criteria taking the judgement of two or more independent reviewers into account and used a flow chart to report search outcomes. However, some crucial methodological shortcomings were found. Practices such as registration of the protocol, reporting the detailed search syntax for one database at least, adherence to reporting guidelines, and assessing the reporting and the methodological quality of primary studies were quite sparse even in recent years. As Pussegoda $e t a l^{4}$ suggested, this fact may be related to the percieved time-consuming task of using guidelines or to the lack of information about the most appropiate tool. According to our data, there is still large room for improvement in the assessment of the methodological quality of included studies in order to attend to Terwee $e t a l$ s warning ${ }^{2}$ of avoiding the risk of presenting biased results, leading to underestimation or overestimation of the quality of an instrument.

Assessment of measurement properties of HRQoL instruments Assessment procedures of measurement properties of HRQoL instruments were diverse. Most of the reviewers used at least one tool. Nevertheless, there were reviewers that applied their own criteria, followed literature recommendations or applied different ad hoc devised checklists. The use of such diverse procedures is noticeable, even in recent years, when well-accepted tools to assess measurement properties are available.

Our meta-review identified up to twelve tools. Seven of them had an itemised structure, offering a comparable approach to rate the evidence on measurement properties. Length and scoring differed, but also the instrument assessment criteria. Actually, depending on the tool used, the approach to assess properties varied greatly, with potentially serious consequences. The fact that a single measurement property is or isn't required can change the status of quality of the evidence supporting the same measurement instrument. The variety of forms found were in concordance to results from related research, which also highlighted the complexity with regard to definitions of measurement properties. ${ }^{6}$ This complexity is also reflected in the search filter developed by the COSMIN initiative. ${ }^{13}$ They recommend using three filters that sum up more than 100 search terms in order to get sensible and specific results. In addition, and also depending on the tool used, other characteristics, such as feasibility, acceptability and burden were assessed. In spite of the diversity, a shared conclusion can be stated as follows: because these instruments are to be used in the daily practice, their usability should be always balanced with other characteristics considered as proper measurement 
properties. ${ }^{39} 40$ For instance, an instrument needs to be long enough to ensure reliability and construct validity, but short enough to ensure the adequate response rate and sample size. Otherwise the instrument intended use and sustainability will be at hazard. ${ }^{39}$

The differences between tools and their potentially serious consequences on the assessment of the quality of the primary studies may be better addressed in the light of three considerations: the date of publication, the main scientific tradition involved when developing the tools, and the intended uses of the instruments under assessment. Some differences can be simply explained by the date of publication of the tools. As an example, where older tools require specific forms of validity evidence related to external variables such as convergent and discriminant validity, recent tools incorporate the more general view of hypothesis testing. That is, when developing a new use for an instrument, hypotheses should be made regarding the expected relations with other relevant variables in their nomological network and these hypotheses and no other should be tested. ${ }^{32}$ Regarding the scientific traditions, the assessment of outcomes is a constitutive part of the disciplines of Education and Psychology where the Testing Standards come from. In these contexts, participation is taken for granted as assessment practices result in high stakes decisions such as, for instance, certification or personnel selection. The main concern regarding integrity of the instrument purpose is its fakeability, which could distort the decision-making process, and this would explain the interest in response processes in this field. ${ }^{41}{ }^{42}$ By contrast, the main objective in the discipline of Medicine is to provide healthcare services. Evaluation of subjective views of patients was a late addition related to the inclusion of HRQoL in the accounting of healthcare outcomes, despite the instruments assessing the patient experience should be acceptable to both patients and clinicians, as Beattie et al highlighted. ${ }^{39}$ Specifically, in the context of disability research, the administrative and respondent burden requires additional consideration. The administrative burden may include the need for a Sign Language interpreter, and the respondent burden includes the length of the questionnaire, which is especially relevant when using HRQoL instruments with cognitively impaired subjects. ${ }^{29}$ Balancing the traditional psychometric criteria, the practicalities of the instruments and patient preferences is a generic recommendation for health research, but becomes a special obligation for research with people with specific needs. ${ }^{29}$ Moreover, devising test accommodations or accessible forms when needed is expected to become a required psychometric criterion in the near future, given that it has already been included under the title 'fairness in testing' as a new section next to validity and reliability in the chapter of measurement foundations in the most recent update of Testing Standards. ${ }^{32}$

Another criterion is that of economic evaluation, traditionally embedded in providing quantitative judgements able to be integrated into mathematical models such as those used in calculating quality-adjusted life years and using preference-based methods to obtain their data. Due to that, some very popular measurement properties such as internal structure based on factor analysis are not relevant and thus not considered in their tools. In this tradition, the main concern regarding the integrity of the instrument purpose is whose values should be considered when determining preferences and how well the preferences of patients and decision makers are likely to conform to the main assumptions of the utility models. $^{2021}$

\section{Intended uses of instruments and their association to measurement properties}

In our view, considering in the first place the intended use of the HRQoL instrument would help to reconcile the different requirements included in each tool. Tools for evaluating the measurement quality of instruments should be adapted or extended according to the different intended uses of these instruments, such as evaluative, impact of disease, analytic, diagnostic, descriptive or predictive. Notice that depending on the intended use of the measure, some domains of validity and reliability may be of greater or lesser relevance. ${ }^{6}{ }^{16}$ For instance, an instrument developed to assess longitudinal changes should demonstrate high responsiveness, ${ }^{6}$ but if used for diagnostic purposes, it should be able to distinguish among individuals or groups, ${ }^{6}$ that is, known groups validity. Another example is the internal consistency reliability based on interitem relationships that may be not relevant for a preference-based instrument but is relevant for an instrument based on a unidimensional measurement model. However, our data showed that only a few authors established a clear link in their recommendations between the intended use of the measure and the reported evidence of measurement properties. The vast field of HRQoL offered a plethora of instruments but, as most reviewers did not take the intended use of the instrument into account, the overall rating of measurement properties was not consistent and thus the instrument may or may not have been adequate for its intended use. Because the evaluation and improvement of quality of life is considered a public health priority, ${ }^{14}$ we strongly encourage researchers to assess the quality of measurement properties of HRQoL instruments according to the intended use of the measure. Otherwise, there is a serious risk of biased results, which could lead to underrating the quality and suitability of the instrument.

\section{CONCLUSIONS}

The quality of the systematic review process has been increasing over time, but it should still improve with regard to the prospective registration of protocol, and with respect to the adoption of guidelines to improve both the methodological and reporting quality of the reviews. In the specific context of systematic reviews of measurement instruments, enhancing the quality of the 
process also involves the assessment of measurement properties by using a standardised tool. The selection of the most suitable tool may be addressed according to the coverage of the appraised measurement properties, but also according to other important criteria, such as the intended use of the HRQoL instruments, the format of the tool and whether it assesses both usability (eg, feasibility or burden) and accommodation (or accessible forms). First, the assessment methodology should be adapted when necessary, establishing the relation between the intended use of the HRQoL instruments and the measurement properties assessed. Second, to standardise the review process, the tool's format should be itemised offering a comparable approach to rate the evidence on measurement properties. Those tools that take the form of guidelines, such as the SACMOT or the economic evaluation would be considerably upgraded if the structure is reconverted, since the current format only allows description rather than critical appraisal of the quality of an instrument, and furthermore, it complicates comparison of results. Lastly, because systematic reviews on measurement properties aim to help professionals to select the best instrument for a clinical scenario, the feasibility, patient's preferences, administrator and respondent burden, and the accommodations (or accessible forms) should be addressed and evaluated. Otherwise the suitability and the intended use of instruments might be compromised, especially in the context of disability research. Tools identified in our meta-review that meet most of these criteria are the COSMIN, EMPRO, SCI criteria, Andresen's tool, CanChild Outcomes and OMERACT, since all of them cover a wide range of measurement properties, offer an item structure, and assess the usability of instruments.

Special mention is due to the COSMIN, the most widespread and comprehensive tool to assess measurement properties of health instruments designed for an evaluative purpose. The COSMIN standards were developed in a Delphy study ${ }^{43}$ aiming to improve the selection of the most appropriate health instrument for a clinical scenario. The most recent version of the COSMIN consists of a manual for conducting systematic reviews of health instruments, providing different steps with respect to the literature search process, the assessment of measurement properties and feasibility of instruments, and the evaluation of the risk of bias ( $\mathrm{RoB})$ of studies according to the Cochrane methodology. ${ }^{16}$ Additionally, the COSMIN initiative recently developed a guideline exclusively focused on assessing the content validity of health instruments, considered the most important property to ensure the adequate reflection of the construct measured. ${ }^{44} 45$ In the light of these considerations, we strongly recommend the application of the latest version of the COSMIN to conduct high-quality systematic reviews on measurement properties of health instruments for an evaluative purpose, or for other purposes with appropriate adaptation.

Despite COSMIN's many strengths, our analysis of the other assessment tools and measurement standards allow us to suggest future lines of work on this tool. First, the current format of COSMIN is fairly complex, requiring high expertise in the field of psychometrics and specific training for its proper application. The reporting of the inter-rater agreement coefficients when reviewers use the last version of COSMIN may provide useful data about its reliability. Second, consideration should be given to the testing standards recommendation on the inclusion of the assessment of fairness (ie, evaluation of accessible forms for specific populations). Third, the feasibility of the measurement instruments, merely described in COSMIN, and their burden, should be properly rated, with examples found in EMPRO or Andresen's tool. Fourth, it must be considered that the RoB evaluation of studies is itself a productive field of research with a long tradition, with specific tools that have been developed for different research questions and study designs. Examples might be found in the Cochrane Collaboration's Tool for Assessing the Risk of Bias of Clinical Trials, ${ }^{46}$ the Newcastle Ottawa Scale ${ }^{47}$ for non-randomised studies, or the Quality Assessment Tool for Cohort Studies. ${ }^{48}{ }^{49}$ From our point of view, the COSMIN proposal could also be simplified and improved by guiding the reviewers towards the identification of the most appropriate RoB assessment tools instead of developing their own RoB appraisal guidelines, taking advantage of knowledge and innovations in that field of research.

And last, but not least, improving the quality of systematic reviews encompasses researchers, sponsors and promoters, but also journals, which should require full compliance with reporting and methodological guidelines, and the use of assessment tools.

Twitter Jaume Vives @VivesJ_Research and Josep-Maria Losilla @jmlosilla Contributors All authors meet the criteria recommended by the International Committee of Medical Journal Editors, ICMJE. All authors made substantial contributions to conception and design, piloted the inclusion criteria and provided the direction of the data extraction and analysis. SL drafted the article and JV, CV and J-ML critically revised the draft for important intellectual content. All authors agreed on the final version.

Funding This work was supported by the Grant PGC2018-100675-B-100, Spanish Ministry of Science, Innovation and Universities (Spain).

Competing interests None declared.

Patient and public involvement Patients and/or the public were not involved in the design, or conduct, or reporting, or dissemination plans of this research.

Patient consent for publication Not required.

Provenance and peer review Not commissioned; externally peer reviewed.

Data availability statement Data are available on reasonable request. Data are available on reasonable request to the authors.

Open access This is an open access article distributed in accordance with the Creative Commons Attribution Non Commercial (CC BY-NC 4.0) license, which permits others to distribute, remix, adapt, build upon this work noncommercially, and license their derivative works on different terms, provided the original work is properly cited, appropriate credit is given, any changes made indicated, and the use is non-commercial. See: http://creativecommons.org/ licenses/by-nc/4.0/.

ORCID iD

Sonia Lorente http://orcid.org/0000-0002-5494-3325 


\section{REFERENCES}

1 Mokkink LB, Terwee CB, Stratford PW, et al. Evaluation of the methodological quality of systematic reviews of health status measurement instruments. Qual Life Res 2009;18:313-33.

2 Terwee CB, Prinsen CAC, Ricci Garotti MG, et al. The quality of systematic reviews of health-related outcome measurement instruments. Qual Life Res 2016;25:767-79.

3 Prinsen CAC, Mokkink LB, Bouter LM, et al. COSMIN guideline for systematic reviews of patient-reported outcome measures. Qual Life Res 2018;27:1147-57.

4 Pussegoda K, Turner L, Garritty C, et al. Identifying approaches for assessing methodological and reporting quality of systematic reviews: a descriptive study. Syst Rev 2017;6:1-12.

5 Pussegoda K, Turner L, Garritty C, et al. Systematic review adherence to methodological or reporting quality. Syst Rev 2017;6:1-14.

6 Rosenkoetter U, Tate RL. Assessing features of psychometric assessment instruments: a comparison of the COSMIN checklist with other critical appraisal tools. Brain Impair 2017:1-16.

7 Reeve BB, Wyrwich KW, Wu AW, et al. ISOQOL recommends minimum standards for patient-reported outcome measures used in patient-centered outcomes and comparative effectiveness research. Qual Life Res 2013;22:1889-905.

8 Lorente S, Vives J, Viladrich C, et al. Tools to assess the measurement properties of quality of life instruments : a meta-review protocol. BMJ Open 2018;8:1-4.

9 Moher D, Liberati A, Tetzlaff J, et al. Preferred reporting items for systematic reviews and meta-analyses: the PRISMA statement. Ann Intern Med 2009;151:264-9.

10 Liberati A, Altman DG, Tetzlaff J, et al. The PRISMA statement for reporting systematic reviews and meta-analyses of studies that evaluate health care interventions: explanation and elaboration. Ann Intern Med 2009;151:W65-94

11 McGowan J, Sampson M, Salzwedel DM, et al. PRESS peer review of electronic search strategies: 2015 guideline explanation and elaboration (PRESS E\&E). Cadth Methods Guidel 2016:40-6.

12 McGowan J, Sampson M, Salzwedel DM, et al. PRESS peer review of electronic search strategies: 2015 guideline statement. J Clin Epidemiol 2016;75:40-6.

13 Terwee CB, Jansma EP, Riphagen II, et al. Development of a methodological PubMed search filter for finding studies on measurement properties of measurement instruments. Qual Life Res 2009;18:1115-23.

14 Secretary's Advisory Committee on National Health Promotion and Disease Prevention Objectives. Health-related quality of life and wellbeing, 2010. Available: https://www.healthypeople.gov/sites/default/ files/HRQoLWBFullReport.pdf

15 Mokkink L, Terwee C, Patrick D, et al. The COSMIN checklist manual, 2012. Available: http://www.cosmin.nl/images/upload/files/COSM INchecklistmanualv9.pdf

16 Mokkink LB, Prinsen C, Patrick D, et al. COSMIN methodology for systematic reviews of patient-reported outcome measures (PROMs). User manual 2018:1-78.

17 Terwee CB, Bot SDM, de Boer MR, et al. Quality criteria were proposed for measurement properties of health status questionnaires. J Clin Epidemiol 2007;60:34-42.

18 Aaronson N, Alonso J, Burnam A, et al. Assessing health status and quality-of-life instruments: attributes and review criteria. Qual Life Res 2002;11:193-215.

19 Lohr KN, Aaronson NK, Alonso J, et al. Evaluating quality-of-life and health status instruments: development of scientific review criteria. Clin Ther 1996;18:979-92.

20 Brazier J, Deverill M, Green C. A review of the use of health status measures in economic evaluation. Health Technol Assess 1999;3:1-164.

21 Brazier J, Ratcliffe J. Measurement and Valuation of Health for Economic Evaluation. In: International encyclopedia of public health. . Elsevier, 2017: Vol. 4. 586-93.

22 Department of Health and Human Services. Guidance for industry patient-reported outcome measures: use in medical product development to support labeling claims: draft guidance. Vol. 20 2006.

23 Department of Health and Human Services. Guidance for industry patient-reported outcome measures: use in medical product development to support labeling claims, 2009.
24 Fitzpatrick R, Davey C, Buxton MJ, et al. Evaluating patient-based outcome measures for use in clinical trials. Vol. 2, 1998.

25 World Health Organization. International classification of functioning (ICF), 2016. Available: www.who.int/classifications/icf/en/ [Accessed 6 Feb 2020].

26 Valderas JM, Ferrer M, Mendívil J, et al. Development of EMPRO: a tool for the standardized assessment of patient-reported outcome measures. Value Health 2008;11:700-8.

27 SCIRE. Spinal cord injury rehabilitation evidence. Available: https:// scireproject.com

28 Johnston MV, Graves DE. Towards guidelines for evaluation of measures: an introduction with application to spinal cord injury. $J$ Spinal Cord Med 2008;31:13-26.

29 Andresen EM. Criteria for assessing the tools of disability outcomes research. Arch Phys Med Rehabil 2000;81:S15-20.

30 Law M. Outcome measures rating form guidelines, 2004. Available: https://www.canchild.ca/system/tenon/assets/attachments/000/000/ 371/original/measguid.pdf

31 OMERACT. Instrument selection for core outcome measurement sets. OMERACT Handbook, 2019. https://omeracthandbook.org/ handbook

32 APA, AERA, NCME. Standards for educational and psychological testing. American Educational Research Association, 2014.

33 APA, AERA, NCME. Standards for educational and psychological testing. American Educational Research Association, 1999.

34 Cook DA, Beckman TJ. Current concepts in validity and reliability for psychometric instruments: theory and application. Am J Med 2006;119:166.e7-166.e16.

35 McDowell I, Spasoff RA, Kristjansson B. On the classification of population health measurements. Am J Public Health 2004:94:388-93

36 Garin O, Ferrer M, Pont A, et al. Disease-specific health-related quality of life questionnaires for heart failure: a systematic review with meta-analyses. Qual Life Res 2009;18:71-85.

37 Speight J, Howarth A. Quality of life in restless legs syndrome: a systematic review of clinical trials and a critical review of instruments. Patient 2010;3:185-203.

38 Chassany O, Holtmann G, Malagelada J, et al. Systematic review: health-related quality of life (HRQOL) questionnaires in gastro-oesophageal reflux disease. Aliment Pharmacol Ther 2008;27:1053-70.

39 Beattie M, Lauder W, Atherton I, et al. Instruments to measure patient experience of health care quality in hospitals: a systematic review protocol. Syst Rev 2014;3:4

40 Lorente S, Losilla J-M, Vives J. Instruments to assess patient comfort during hospitalization: a psychometric review. J Adv Nurs 2018;74:1001-15.

41 Ferrando PJ, Anguiano-Carrasco C. A structural model-based optimal Person-Fit procedure for identifying Faking. Educ Psychol Meas 2013;73:173-90.

42 Ferrando PJ, Anguiano-Carrasco C. A structural equation model at the individual and group level for assessing faking-related change. Struct Equ Model 2011;18:91-109.

43 Mokkink LB, Terwee CB, Patrick DL, et al. The COSMIN checklist for assessing the methodological quality of studies on measurement properties of health status measurement instruments: an international Delphi study. Qual Life Res 2010;19:539-49.

44 Terwee CB, Prinsen CA, Chiarotto A. COSMIN methodology for assessing the content validity of PROMs: user manual. Vol. 120, 2018.

45 Terwee CB, Prinsen CAC, Chiarotto A, et al. COSMIN methodology for evaluating the content validity of patient-reported outcome measures: a Delphi study. Qual Life Res 2018;27:1159-70.

46 Higgins JPT, Altman DG, Gøtzsche PC, et al. The Cochrane collaboration's tool for assessing risk of bias in randomised trials. BMJ 2011;343:d5928-9.

47 Wells G, Shea B, O'Connell D, et al. The Newcastle-Otawa scale (NOS) for assessing the quality of nonrandomised studies in meta-analyses, 2000. Available: www.ohri.ca/programs/clinical epidemiology/oxford.asp

48 Jarde A, Losilla J-M, Vives J, et al. Q-Coh: A tool to screen the methodological quality of cohort studies in systematic reviews and meta-analyses. Int J Clin Heal Psychol 2013;13:138-46.

49 Jarde A, Losilla J-M, Oliveras I. Quality assssment tool for cohort studies (Q-COH II) user's manual, 2014: 1-13. 